\title{
Le système éducatif du Cap Vert : réformes et enjeux actuels
}

\section{Ana Cristina Pires Ferreira}

\section{(2) OpenEdition}

1 Journals

Édition électronique

URL : http://journals.openedition.org/ries/3458

DOI : 10.4000/ries.3458

ISSN : 2261-4265

Éditeur

Centre international d'études pédagogiques

Édition imprimée

Date de publication : 1 septembre 2013

Pagination : 21-27

ISBN : 978-2-85420-600-5

ISSN : 1254-4590

\section{Référence électronique}

Ana Cristina Pires Ferreira, «Le système éducatif du Cap Vert : réformes et enjeux actuels », Revue internationale d'éducation de Sèvres [En ligne], 63 I septembre 2013, mis en ligne le 01 septembre 2015, consulté le 30 avril 2019. URL : http://journals.openedition.org/ries/3458 ; DOI : 10.4000/ries.3458

Ce document a été généré automatiquement le 30 avril 2019

(c) Tous droits réservés 


\title{
Le système éducatif du Cap Vert : réformes et enjeux actuels
}

\author{
Ana Cristina Pires Ferreira
}

1 Le Cap Vert, archipel de 491575 habitants (2010), à $450 \mathrm{~km}$ du Sénégal en Afrique occidentale, est marqué par une histoire de métissage ${ }^{1}$ et d'extraversion sociale (l'émigration) et économique (participation au commerce des esclaves, dépendance de l'aide internationale).

2 Dans un contexte de sécheresses répétées dues à la proximité du désert du Sahara et à la rareté des ressources naturelles, l'émigration devient une stratégie de survie de la population. L'émigration est donc une caractéristique importante du pays par sa dimension (le nombre d'expatriés dépasse celui des autochtones) et par son poids financier - les envois des émigrés représentaient environ un tiers du PIB par habitant (3 222 \$ US), en $2009^{2}$.

3 La dépendance économique et la pauvreté sont autant de vulnérabilités qui, associées à la politique menée (non alignement et bonne gouvernance), ont fait du Cap Vert, après son indépendance en 1975, un donor darling, jusqu'à son accession, en 2007, au groupe des pays à revenu moyen (976 à 3855 \$ US/hab.).

4 L'économie du Cap Vert est dominée par le secteur des services (65\% du PIB) ${ }^{3}$ notamment, le tourisme. Néanmoins, la fonction publique d'État est toujours le premier secteur d'emploi et l'éducation y occupe une place prépondérante.

5 L'éducation devient, après l'indépendance, un moyen de construction nationale et de transformation du pays. Elle est aussi un moyen d'ascension sociale et d'amélioration des conditions de vie de la population. D'où l'investissement du gouvernement, de la société et des organisations internationales éducatives et d'aide au développement dans le sens « d'une école de masse ». Ainsi, en $2010 / 2011^{4}$, un tiers de la population nationale était scolarisé (145 416 élèves) dont $16 \%$ dans le préscolaire, $48 \%$ dans l'enseignement de base et $37 \%$ dans le secondaire, sans disparité entre les sexes. Les individus possédant un niveau d'études supérieures représentaient $5 \%$ de la population totale et les analphabètes $12,5 \%{ }^{5}$. 
6 Sur la même période, les élèves étaient encadrés, par 6875 enseignants, féminisés à $63 \%$ et possédant, pour la majorité, une formation spécifique. Le Cap Vert, comme d'autres pays, suit le mouvement de "l'universitarisation » de la formation des enseignants ${ }^{6}$, ce qui demande une évolution de leur statut et renvoie à l'enjeu de revalorisation de la carrière enseignante.

7 Malgré ses vulnérabilités, le Cap Vert est sur la voie du développement, du progrès et de la reconnaissance internationale ; l'éducation y contribue fortement.

\section{L'administration, la structure du système éducatif et les enjeux actuels}

8 Les objectifs généraux du système éducatif traduits dans la Loi de base du système éducatif (LBSE) en 1990, et repris dans sa nouvelle version en 2010, sont : « la formation intégrale de l'individu dans une perspective universaliste, l'articulation étroite entre la formation et le travail, la préservation de l'identité nationale et l'élimination de l'analphabétisme $»^{7}$. Qu'impliquent-ils dans la structure du système éducatif ? Quels en sont les enjeux?

9 Le système éducatif est national et relève du ministère de l'Éducation (ME) et du ministère de l'Enseignement supérieur et de la Science. La configuration actuelle, malgré quelques ajustements, date des années 1990, où fut opérée une restructuration par une réforme d'envergure. On dénombre trois sous-systèmes: le préscolaire; le scolaire concernant l'enseignement de base, universel, gratuit et obligatoire (6-12 ans) et l'enseignement secondaire, d'une durée de six années; et l'enseignement supérieur (universités et instituts supérieurs). Le système intègre aussi une composante extrascolaire vouée à l'éducation des adultes (à partir de 15 ans), articulant une formation générale de base (trois phases) et une formation professionnelle. Depuis cinq ans, grâce aux progrès de l'alphabétisation ${ }^{8}$ et de par les -exigences économiques, l'enseignement « de seconde chance », pour les jeunes qui n'ont pas été scolarisés ou qui ont abandonné l'école, se développe.

10 Le préscolaire (4-6 ans) est facultatif et vient en complément de l'éducation familiale. Il n'est pas pris en charge par le gouvernement ; c'est une politique qui perdure depuis l'époque coloniale. Le ME assure le rôle de tutelle pédagogique mais la création et la gestion des établissements reviennent aux municipalités, aux ONG et au privé. Une discussion sur l'éducation de base est en cours et l'enjeu est d'y intégrer le préscolaire pour réduire les inégalités scolaires et sociales qui influencent négativement les apprentissages.

11 L'enseignement de base et l'enseignement secondaire sont divisés en cycles de deux ans chacun en fonction desquels le curriculum formel est défini avec une diversification des cursus (général et technico-professionnel) dans le $3^{\mathrm{e}}$ cycle du secondaire.

12 L'enseignement supérieur a évolué d'une formation technique post-secondaire (trois ans) publique, dans les années 1990, à une formation polyvalente essentiellement privée, dans les années $2000^{9}$. L'offre est concentrée sur les deux îles plus peuplées (Santiago et São Vicente) et réservée aux plus aisés, ce qui reproduit les inégalités sociales et ne permet pas de répondre réellement aux besoins du pays. 
13 Le système éducatif englobe aussi un curriculum national (plan d'études, programmes, système d'évaluation) qui est en cours de refonte et vise à la formation d'un nouveau profil du citoyen cap-verdien ${ }^{10}$. Il comprend cinq matières fondamentales pour tous (langue portugaise, mathématiques, sciences, éducation physique et à la citoyenneté). Dans l'enseignement de base, ces matières étaient enseignées par un seul professeur, jusqu'en 2009. Les examens ont été abolis à la fin des années 1990. L'enseignement se fait en portugais, dans un contexte de diglossie et d'un rapport problématique avec la langue nationale, le créole cap-verdien. Les enjeux sont de dépasser les tensions politiques et sociales (crainte d'isolement, de renforcement d'inégalités) et de formaliser son utilisation dans les écoles en référence aux objectifs de la politique éducative : renforcer l'identité et la culture nationales et améliorer la qualité des apprentissages (LBSE, 2010). Mais comment les mettre en œuvre? Avec quelles ressources et quelles stratégies?

Le système éducatif se déploie sur le territoire national au travers des structures déconcentrées dans chaque unité territoriale (concelho), des pôles éducatifs ${ }^{11}$ et des écoles secondaires, au niveau local. Malgré quelques initiatives de décentralisation à la fin des années 1990, le système éducatif reste centralisé. Les enjeux de contrôle et de régulation de l'administration par l'État restent de mise, surtout pour préserver l'image internationale de « bonne gouvernance » et les flux d'investissements dans le pays.

\section{Un fonctionnement du système éducatif au gré de réformes}

15 La politique d'éducation, telle un programme d'action (Meny \& Thoenig, 1989), vise essentiellement à résoudre, depuis l'indépendance, des problèmes d'analphabétisme, de besoins de main d'œuvre pour le développement du pays, de construction et de préservation de l'identité nationale. L'enjeu était, comme pour d'autre pays récemment indépendants, de mettre en place un nouveau système éducatif adapté au contexte d'une nation naissante et dans une logique de développement, d'où une succession de réformes en tant qu'outils de politique publique pour changer durablement l'organisation et le fonctionnement du système éducatif.

16 Trois grandes périodes de réformes éducatives peuvent être identifiées ${ }^{12}:$ les premières années de l'indépendance (1975-1985), les années 1990 (1990-2002) et la période de 2003 à nos jours. Elles révèlent des façons différentes de produire et mettre en œuvre les politiques éducatives de l'État ainsi que l'influence de politiques éducatives transnationales.

17 Pendant la première période, la rhétorique de changement, notamment dans le sens de la nationalisation et de l'africanisation du système, est plus importante que la pratique. L'objectif de transformation du système éducatif échoue car les enjeux d'affirmation du Parti-État et de la survie immédiate de la population prévalent et les partenaires techniques et financiers internationaux s'intéressent peu à l'éducation.

18 La persistance des principaux problèmes de l'éducation conduisent à une réforme d'envergure dans les années 1990, pour moderniser et restructurer le système éducatif. Demeure la préoccupation de la nationalisation du système mais celle d'africanisation disparaît. Cette réforme s'est construite à la croisée des aspirations nationales mentionnées et du mouvement international sur l'éducation pour tous (Jomtien, 1990). De 
cette réforme résulte la massification de l'accès à l'éducation de base et l'expansion de l'enseignement secondaire.

Quelques données statistiques ${ }^{13}$ permettent d'illustrer les progrès accomplis par l'éducation de base pour tous mais elles révèlent aussi le déficit d'efficacité interne. Le taux brut de scolarisation est passé de $131 \%$ en 1990 à $123 \%$ en 2000, puis à $109 \%$ en 2010. Le taux de redoublement qui était à $18 \%$ en 1990 baisse à $12 \%$ en 2000. En 2009, il concernait $10,7 \%$ des élèves.

Pour l'enseignement secondaire, le taux brut de scolarisation est passé de $63,5 \%$ en 2000 à $85,3 \%$ en $2010^{14}$. Ces données démontrent les répercussions de la massification de l'enseignement de base dans les années 1990 sur l'enseignement secondaire. Ce phénomène touche aussi les taux de redoublement et d'abandon. Le premier est quasi constant entre $2000(20,5 \%)$ et $2009(19,5 \%)$, tandis que le deuxième régresse légèrement entre $2000(9,4 \%)$ et $2009(8 \%)^{15}$.

21 Le diagnostic de la situation fait par le ME, lorsque qu'il a entamé le processus de révision du curriculum en $2006^{16}$, montre aussi l'accentuation des problèmes signalés dans le Plan stratégique de l'Éducation (PSE), 2003-2013, à savoir :

- «le rapport problématique entre la langue nationale (le créole cap-verdien) et la langue officielle, véhiculaire (le portugais) » se traduit notamment, par l'inefficacité de la méthode d'enseignement de cette dernière et par son influence sur le rendement des élèves ;

- «la persistance de pratiques privilégiant la mémorisation»;

- «la faible pertinence sociale et économique des apprentissages surtout dans l'enseignement secondaire », par rapport au profil de citoyen souhaité : un Cap-verdien conscient de son identité nationale, compétent et qui s'adapte au contexte local, national et global en mutation $^{17}$. Ce profil doit répondre, d'une part, à l'aspiration nationale à plus de prospérité, à la volonté politique d'insertion du pays dans l'économie globale et aux dynamiques sociales (notamment, plus d'affirmation de la femme et de son rôle social et économique, de développement d'une classe moyenne, d'une certaine violence urbaine); d'autre part, répondre aux injonctions internationales actuelles d'adaptabilité et d'employabilité.

La démocratisation de l'accès à l'éducation s'accompagne donc d'une mise en évidence de problèmes qui appellent à de nouveaux changements dans l'organisation et le fonctionnement du système éducatif. Ainsi, une nouvelle restructuration des cycles d'enseignement de base et secondaire est en perspective (LBSE, 2010). Les buts sont d'allonger l'enseignement de base obligatoire à huit années d'études, d'ici 2016, puis à dix années d'études, en 2020. Il s'agit de légitimer une politique de l'éducation au service du développement économique, de meilleure qualification de la main d'œuvre pour une économie plus compétitive, mais aussi de satisfaire une demande sociale qui valorise les diplômes en tant que moyen d'ascension sociale. La restructuration mentionnée est l'un des principaux pans de la nouvelle réforme éducative, qui vise à : "répondre de façon adéquate aux défis globaux de la société cap-verdienne ; améliorer le fonctionnement et moderniser le système éducatif » (LBSE, 2010). Cette politique s'inscrit dans le projet de transformation du pays pour une insertion compétitive dans l'économie mondiale dont la vision est: « une nation inclusive, prospère, avec des opportunités pour tous (...), une économie dynamique et innovatrice $»^{18}$. Ces orientations associées aux principes de la restructuration du système scolaire à savoir, "l'universalité de l'accès, l'équité et la durabilité financière ${ }^{19}$ ne sont pas simples à mettre en œuvre, tant les logiques qui les sous-tendent sont différentes et en tension. Comment concilier l'égalité dans l'accès à l'éducation et l'équité, qui demande une discrimination positive individuelle, la 
compétitivité économique (s'appuyant sur l'individu) et la promotion de la cohésion et de la justice sociale (s'appuyant sur le collectif)?

La recherche d'une rationalisation de l'organisation et du fonctionnement du système éducatif est un enjeu actuel avec l'introduction des nouvelles technologies d'information et de communication dans la gestion du système à différents niveaux et la publication d'une nouvelle Loi de bases du système éducatif (2010). Celle-ci doit permettre un nouveau cycle de réforme du système éducatif qui, de fait, est en cours depuis 2006 avec la refonte du curriculum orientée vers les compétences et qui semble poser déjà la question de sa faisabilité, avec les difficultés rencontrées dans la phase d'expérimentation des nouveaux programmes.

Si l'on considère les réformes du système éducatif comme des processus de décisions politiques et institutionnelles, que nous apprennent-elles des modes d'action de l'État? La réforme éducative des années 1990, a suivi un processus organisé et centralisé, de planification a priori intégrée dans des plans de développement quinquennaux et selon l'approche projet avec des partenaires techniques et financiers internationaux (Banque mondiale et Banque africaine de développement) très prégnants. Les décisions ont été prises de façon pragmatique dans des jeux d'intérêts souvent divergents entre acteurs internationaux (experts techniques internationaux, coordinateurs de projets) et acteurs nationaux (dirigeants et cadres du ME et gestionnaires de projet). Les premiers visaient l'efficacité de l'investissement, la diffusion, voire l'imposition d'un modèle d'école (moderne, centrée sur les élèves et sur la qualité des apprentissages) et de développement économique, alors que les deuxièmes cherchaient à traduire les politiques transnationales et à sauvegarder une certaine autonomie.

Le Cap Vert : principales données

\begin{tabular}{|c|c|}
\hline Habitants $(2010)^{\star *}$ & 491575 \\
\hline Indice de développement humain (PNUD) $(2010) * *$ & 0,566 \\
\hline Espérance de vie (moyenne, 2010)** & 70,8 ans \\
\hline PIB par habitant (2009) & $3222 \$$ US \\
\hline Taux de chômage $(2011) * *$ & $12,2 \%$ \\
\hline Taux de chômage des jeunes de 15-24 ans (2011) ** & $27,1 \%$ \\
\hline Indice d'incidence de la pauvreté (2007) ** & $26,6 \%$ \\
\hline Taux d'analphabètes avec 15 ans et plus (2010) ** & $12,5 \%$ \\
\hline Taux brut de scolarisation dans l'enseignement de base (2011) * & $109 \%$ \\
\hline Taux de redoublement dans l'enseignement de base (2009)* & $10,7 \%$ \\
\hline Taux brut de scolarisation dans l'enseignement secondaire $(2011)^{*}$ & $85,3 \%$ \\
\hline Taux de redoublement dans l'enseignement secondaire (2009)* & $19,5 \%$ \\
\hline Pourcentage du budget de l'Éducation par rapport au PIB (2009) ${ }^{\star * *}$ & $7 \%$ \\
\hline
\end{tabular}

* Source : Ministère de l'Éducation du Cap Vert - RESEN

** Source : Institut national de statistique (http//www.ine.cv)

*** Source : Ministère de l’Éducation, PNUD Cap Vert

Néanmoins, après l'évaluation du cycle de réforme et l'élaboration du PSE (2003-2013), le mode de fonctionnement décrit connaît quelques changements. Il ne s'agit plus de réformes d'envergure, dans le sens top-down mais d'initiatives diverses d'innovation partant des services centraux du ministère de l'Éducation et des écoles, dans le cadre de coopérations bilatérales. Les organisations internationales continuant à assurer leur rôle 
de promoteurs d'innovations, le jeu d'acteurs est brouillé par une logique de concurrence. On note par exemple, par rapport à 1990, une faible participation d'institutions portugaises dans la refonte du curriculum scolaire et plus d'institutions francophones (Organisation international de la francophonie). D'autres acteurs, comme les ONG nationales, gagnent de la visibilité. Le caractère "ordonné » de la réforme des années 1990 a cédé la place à la diversité des initiatives. En fait, il y a un processus de «prêt » et d'«emprunt " de politiques entre les pays et certains jouent un rôle de "laboratoire politique $»^{20}$, le Cap Vert en est un.

\section{BIBLIOGRAPHIE}

BAYART J.-F. (1999) : «L'Afrique dans le monde : une histoire d'extraversion ». Critique internationale. Vol. 5, p. 97-120.

CABO Verde (2011) : Ministério da Edu-cação e Desporto. Relatório do Estado do Sistema Educativo Nacional.

MENY Y. \& THOENIG J.-C. (1989) : Politiques Publiques. Paris : Presses Universitaires de France.

PIRES-FERREIRA A. C. (2012) : La gestion de l'éducation au Cap Vert : prises de décision, participation et autonomie. Saarbrücken : Éditions universitaires européennes.

TEHIO V. \& CROS F. (Éd.) (2009) : Politiques publiques en éducation : l'exemple des réformes curriculaires. Actes du Séminaire final sur l'étude des réformes curriculaires par l'approche par compétence en Afrique. Paris : CIEP/OIF/AFD/BAD/Ministère des Affaires étrangères et européennes.

VAN ZANTEN A. (2011) : Les Politiques d'éducation. Paris : Presses universitaires de France.

\section{NOTES}

1. Le processus de métissage a débuté tôt, suite à la découverte de l'archipel par le Portugal, en 1460 , et à son peuplement avec des esclaves amenés des pays africains voisins et des Portugais, ainsi que d'autres Européens, à partir de 1462.

2. Sources : Institut national de statistique et ministère des Finances.

3. Ministère du Plan du Cap Vert. Document de croissance et de réduction de la pauvreté (II), 2008.

4. Ministère de l'Éducation. Annuaire statistique de l'éducation 2010-2011.

5. http//www.ine.cv/dadostats/dados.aspx ?d=1, consulté le 8 juin 2013.

6. Décret-Loi $\mathrm{n}^{\circ} 17 / 2012$, du 21 juin, qui transforme l'Institut pédagogique, institution non supérieure pour la formation des professeurs de l'enseignement de base en Institut universitaire d'éducation.

7. Décret-loi n 272010 du 7 mai, qui revoit les bases du système éducatif, article 5 .

8. Le taux d'alphabétisation est passé de $60 \%$ en 1975 à $87 \%$, en 2010 , pour la population de 15 ans et plus (Recensement de la population, 2010).

9. La première université a été créée en 2001 par initiative privée ; la seule université publique existante a été mise en place en 2006. En 2012, dix institutions sur douze étaient privées. 
10. Décret-loi $n^{\circ} 32 / 2009$ du 14 septembre sur les nouveaux plans d'études.

11. Groupement d'écoles d'enseignement de base.

12. Périodisation fondée sur des événements marquants : la première révision du curriculum (1975-1976) ; la publication de la première Constitution de la République indépendante (1980) ; le projet Éducation I (1986 à 1988), financé par la Banque mondiale, d'élaboration d'études prospectives pour la réforme des années 1990 ; la publication de la première Loi de bases du système éducatif (1990) et sa mise en œuvre par une succession de projets et l'évaluation de la réforme des années 1990 (2000-2002); l'élaboration du Plan stratégique de l'éducation (2003-2013).

13. Sources: Rapport sur l'état du système éducatif national élaboré par le ministère de l'Éducation en 2011 (RESEN) ; UNESCO, http//stats.uis.unesco.org/unesco pour les données plus récentes; pour les données de 1990, les thèses de doctorat d'Ana -Ferreira, La Gestion de l'Éducation au Cap Vert, 2004 et d'Alcides Moura, Eficácia social (qualidade) do -Sistema educativo em Cabo Verde, 2009.

14. Ministère de l'éducation, RESEN, 2011, p. 77.

15. Op.cit., p 121.

16. Unité de développement du curriculum. Document d'orientation de la révision du curriculum, 2006.

17. LBSE, 2010, qui reprend les mêmes objectifs et principes que celle de 1990 (articles 8, 9 et 10).

18. Programme du gouvernement 2011-2016, p. 16.

19. Préambule de la LBSE, 2010.

20. Steiner-Khamsi, (2004), cité par Zaten, 2011, p. 62.

\section{INDEX}

Mots-clés : politique éducative, réforme de l'enseignement, système éducatif

Index géographique : Cap Vert

Keywords : educational policy, educational reform, educational system

Palabras claves : política educacional, reforma de la educación, sistema educativo

\section{AUTEUR}

\section{ANA CRISTINA PIRES FERREIRA}

Ana Cristina Pires Ferreira est doctorante au département des sciences sociales et humaines de l'Université du Cap-Vert. 